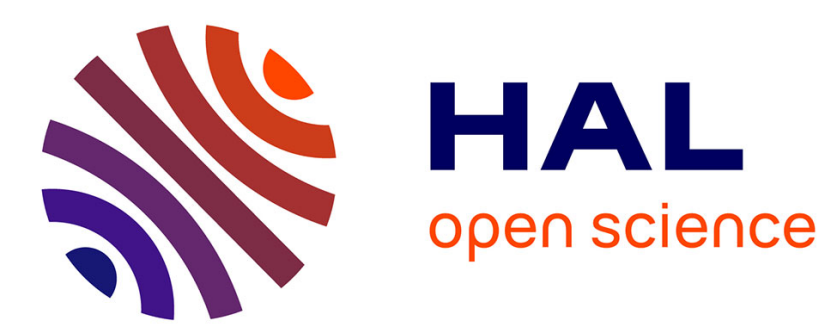

\title{
Needle Steering Fusing Direct Base Manipulation and Tip-based Control
}

\author{
Jason Chevrie, Alexandre Krupa, Marie Babel
}

\section{To cite this version:}

Jason Chevrie, Alexandre Krupa, Marie Babel. Needle Steering Fusing Direct Base Manipulation and Tip-based Control. IEEE Int. Conf. on Robotics and Automation, ICRA'16, May 2016, Stockholm, Sweden. hal-01304860

\section{HAL Id: hal-01304860 \\ https://hal.inria.fr/hal-01304860}

Submitted on 20 Apr 2016

HAL is a multi-disciplinary open access archive for the deposit and dissemination of scientific research documents, whether they are published or not. The documents may come from teaching and research institutions in France or abroad, or from public or private research centers.
L'archive ouverte pluridisciplinaire HAL, est destinée au dépôt et à la diffusion de documents scientifiques de niveau recherche, publiés ou non, émanant des établissements d'enseignement et de recherche français ou étrangers, des laboratoires publics ou privés. 


\title{
Needle Steering Fusing Direct Base Manipulation and Tip-based Control
}

\author{
Jason Chevrie ${ }^{1}$, Alexandre Krupa ${ }^{2}$, Marie Babel $^{3}$
}

\begin{abstract}
Works on robotic needle steering often consider either tip-based control of flexible beveled tip needles or control of the bending of symmetric tip needles. In this paper a control law for needle steering which uses both direct manipulation of the needle base and the control of the tip deflection, is proposed. A 3D model of a beveled tip needle is used to determine the base motion that is needed to obtain the desired tip motion. The needle-tissue interaction model uses local virtual springs placed along the needle shaft and takes into account the interaction forces at the bevel. Online estimation and update of this model parameters is performed via visual feedback. The low level controller uses the task function framework to allow control of the tip velocity. Additionally duty cycling method is used if a reduction of the natural deflection of the needle tip is needed. Finally an experimental targeting task in a gelatin phantom is presented for preliminary validation of the framework. The method provided sub-millimeter accuracy on a target that would be unreachable using only tip-based control.
\end{abstract}

\section{INTRODUCTION}

Surgical interventions using needles have been increasingly used over the last decades. One well-known advantage of needles is the reduced trauma that they cause in the tissue. This allows non-invasive operations, thus reducing the recovery time needed after the operation. However, accurate needle steering in soft tissue remains a challenging task for the clinicians. Indeed, this surgical act is sensitive to any deformations of the tissues, target displacements or needle bending. At the same time the clinician has reduced visibility since no direct vision is possible. Naturally, the effect of mistargeting can be traumatic. It is typically the case in brachytherapy, where misplacement of the radioactive seeds can cause the destruction of healthy tissues. Robot assisted procedures can thus be a great help in this task and have been an active research field for many years [1].

In this context, the bending of the needle was at first considered a drawback that had to be reduced. Indeed, the steering of a flexible needle requires from the clinician an even more accurate comprehension of the interaction between the needle and the tissues [1]. However, it has then been shown that using flexible needles could allow target reaching that would otherwise be unreachable by a straight line [2]. A robotic assistance of this challenging task is thus even more necessary.

Pioneer work was conducted by DiMaio et al. [3] on flexible needle steering in soft tissues. A finite element model was

\footnotetext{
${ }^{1}$ Jason Chevrie is with Université de Rennes 1 and IRISA, France, jason.chevriedirisa.fr

${ }^{2}$ Alexandre Krupa is with Inria Rennes - Bretagne Atlantique and IRISA, France, alexandre.krupa@inria.fr

${ }^{3}$ Marie Babel is with Insa Rennes and IRISA, France, marie.babeleirisa.fr
}

used to model both needle and tissues. The needle motion and tissue deformations could be predicted when the needle was moved by its base. Nevertheless, real time modeling could not be achieved because of the model complexity. A simpler framework was proposed by Glozman et al. [4][5]. The needle-tissue interaction was there modeled only locally with virtual springs placed along the needle shaft. In both methods the needle tip trajectory is controlled by using the transverse motion of the base to deform the tissues and bend the needle. This provides a good steerability at the beginning of the insertion (low depth in tissue). However it can result in large tissue stress and possible tissue damage once the needle is deeply inserted.

From the observation that a beveled needle tends to bend when inserted [6], most recent works mainly focus on the steering of very flexible needles using this property. It has been shown by Webster et al. [2] that the trajectory of the needle tip could be modeled by simple kinematic models, such as the unicycle or bicycle ones. Many control schemes have then been proposed using these models. In [7] sliding mode control is proposed such that the bevel is always oriented toward the target. Hauser et al. [8] use the helical trajectories obtained when simultaneously inserting and rotating the needle. When the rotation speed is large with respect to the insertion speed, straight trajectories can be obtained. The duty cycling control method [9] allows a linear control of the effective curvature of the trajectories. This facilitates the reduction of the complexity of some high level planners, such as the Rapidly exploring Random Tree planner [10]. The method was used with an online estimation of the natural curvature for a targeting task in [11].

The basic assumption when using the kinematics models is that the needle shaft exactly follows the tip trajectory. This is the case when using a very flexible needle embedded in a hard medium. In practice, however, the tissue can be rather soft and the needles commonly used by clinicians not completely flexible. Misra et al. [12] proposed a complete mechanics based model to predict the tip deflection when the needle is inserted. Contrary to the kinematics models, this model takes into account the tissue deformations due to the bending of the needle inside the tissue. In [13] this model was simplified by adding the same virtual springs approach as in [4] and used to control the needle trajectory.

Our first contribution in this paper is the design of a model that simulates the evolution of a beveled tip needle in $3 \mathrm{D}$ space and in real time. The needle tissue interaction is modeled by using an extension of the virtual springs model [4] to 3D. A similar bevel model to the one proposed in [12] is added to take into account the force generated at the 
needle tip during insertion. The model we proposed is then used to derive the relationship between the motion of the tip and the motion of the base of the needle.

The second contribution is a new control law based on the proposed needle model. It allows the steering of the needle tip toward a desired direction. The originality of our approach is to use transverse motion control to increase the reachable region and to switch to a duty cycling method when a reduction of the natural curvature is needed, i.e when rather straight trajectories must be achieved. For the rotation along the shaft, we use sliding mode control [7] to orient the bevel in such a way that the needle tends to bend in the desired direction. We also added a secondary task to reduce the tissue deformations when possible.

The paper is organized as follows: our 3D model of the needle is presented in section II. In section III we present the different parts of our control law. Our experimental setup and results are then shown in section IV. Finally, conclusions and future works are shown and discussed in section $\mathrm{V}$.

\section{NEEDLE MODELING WITH VIRTUAL SPRINGS}

In this section we introduce the model that we use to estimate the behavior of the needle when the motion of the base is controlled. The model proposed by Glozman et al. [4] is first extended: modifications are made in such a way that high bending angles of a 3D needle can be modeled. This allows the modeling of highly flexible needles trajectories in space. The tip model proposed by Misra et al. [12] is also combined to consider the case of a beveled tip.

\section{A. Spring Model}

The interaction between the needle and the tissue is modeled locally with $N$ virtual springs placed all along the needle shaft. Each spring $i$ is defined by a rest position $\boldsymbol{p}_{0, i}$, a stiffness $K_{i}$ and a plane $P_{i}$ that contains $\boldsymbol{p}_{0, i}$ (see Fig. 1a). The rest position of the spring corresponds to the rest location of the tissue if no needle is pushing on it. Each spring exerts a force on the needle at the location where the needle crosses the plane $P_{i}$. We use here the model in a quasi-static case. So we assume that the frictional part, that is tangential to the needle, can be neglected. The springs are only used to model the normal forces $\boldsymbol{F}_{s, i}$ applied on the needle shaft. For this, the orientation of the planes $P_{i}$ are constantly updated such that the exerted force stays normal to the needle. The force can thus be expressed according to

$$
\boldsymbol{F}_{s, i}=K_{i}\left(\boldsymbol{p}_{n, i}-\boldsymbol{p}_{0, i}\right),
$$

with $\boldsymbol{p}_{n, i}$ the point of the needle which crosses the plane $P_{i}$.

We consider here that the tissue stiffness per unit length $K_{T}$ around the needle is constant all along the needle. The stiffness of each spring can thus be adapted in such a way that the equivalent tissue stiffness per unit length remains the same all along the needle, that is

$$
K_{i}=K_{T} l_{i}
$$

where $l_{i}$ is the length of the needle that is supported by the spring $K_{i}$.

\section{B. Bevel tip}

As proposed by Misra et al. in [12], we choose here to model the efforts exerted at the tip with triangular loads distributed on each side of the tip. Let $\alpha$ be the bevel angle, $\beta$ the cut angle, $b$ the bevel length, $a$ the length of the bottom edge of the needle and $O$ the center of the needle located just before the bevel (see Fig. 1b). The normal force $F_{t i p}$ and moment $M_{t i p}$ exerted at the point $O$ can be expressed as

$$
\begin{aligned}
F_{t i p} & =\frac{K_{T} a^{2}}{2} \tan \beta-\frac{K_{T} b^{2}}{2} \tan (\alpha-\beta) \cos \alpha \\
M_{t i p}= & -\frac{K_{T} a^{3}}{6} \tan \beta \\
& +\frac{K_{T} b^{2}}{2} \tan (\alpha-\beta)\left(\frac{a}{3} \cos (\alpha)-\frac{b}{6} \sin (\alpha)^{2}\right) .
\end{aligned}
$$

Knowing the orientation of the base frame $\mathcal{F}_{b}$ (see Fig. 1a), the orientation of the tip frame $\mathcal{F}_{t}$ around the tip axis can be deduced. We choose here to assume that the torsional bending of the needle can be neglected. This way, we can obtain the orientation of $\mathcal{F}_{t}$ by integrating the local rotation around the out of plane vector all along the needle.

\section{Needle Model}

The needle is modeled by a succession of $N+1$ segments such that the extremities of the segments lay on the planes of the virtual springs, except for the needle base that is fixed and the needle tip that is free. Each segment is approximated in $3 \mathrm{D}$ using a polynomial curve $\boldsymbol{S}_{i}(l)$ of order $n$ so that

$$
\boldsymbol{S}_{i}(l)=\boldsymbol{M}_{i}\left[\begin{array}{llll}
1 & l & \ldots & l^{n}
\end{array}\right]^{T},
$$

where $i \in[1, N+1]$ is the segment index and $\boldsymbol{S}_{i}(l) \in \mathbb{R}^{3}$ is the position of a point of the segment at the curvilinear coordinate $l \in\left[0, L_{i}\right]$ with $L_{i}$ the total length of the segment. $M_{i} \in \mathbb{R}^{3 \times(n+1)}$ is a matrix containing the coefficients of the polynomial curve. In our case, real-time performance is expected: so the model complexity should be low. A third order polynomial is thus chosen, since it is the lowest sufficient order for which the mechanical equations can directly be solved.

To maintain adequate continuity properties of the needle, second order continuity constraints are added, namely defined as

$$
\begin{aligned}
\boldsymbol{S}_{i}\left(L_{i}\right) & =\boldsymbol{S}_{i+1}(0), \\
\left.\frac{\mathrm{d} \boldsymbol{S}_{i}}{\mathrm{~d} l}\right|_{l=L_{i}} & =\left.\frac{\mathrm{d} \boldsymbol{S}_{i+1}}{\mathrm{~d} l}\right|_{l=0}, \\
\left.\frac{\mathrm{d}^{2} \boldsymbol{S}_{i}}{\mathrm{~d} l^{2}}\right|_{l=L_{i}} & =\left.\frac{\mathrm{d}^{2} \boldsymbol{S}_{i+1}}{\mathrm{~d} l^{2}}\right|_{l=0} .
\end{aligned}
$$

The total normal force $\boldsymbol{F}_{i}$ at the extremity of the segment $i$ can be calculated from the sum of the forces exerted by the springs located from this extremity to the needle tip, so that

$$
\boldsymbol{F}_{i}=\Pi_{i}\left(F_{t i p} \boldsymbol{y}_{t i p}+\sum_{j=i}^{N} \boldsymbol{F}_{s, j}\right),
$$




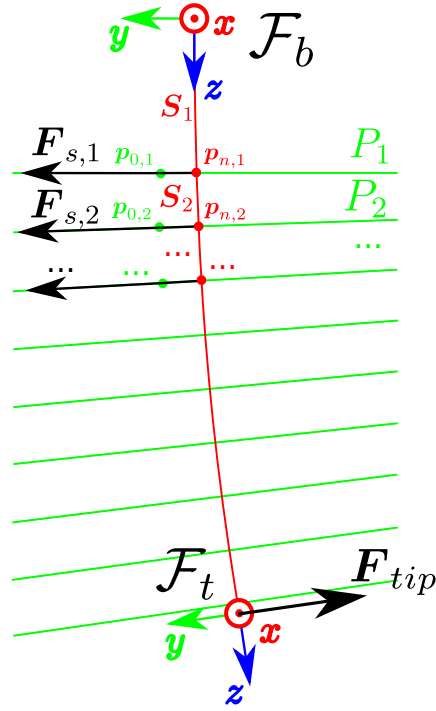

(a) 2D projection of the needle

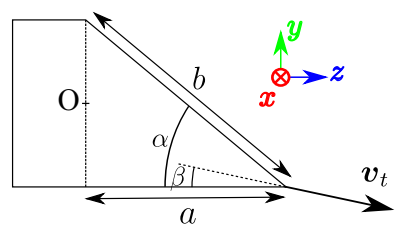

(b) Beveled tip

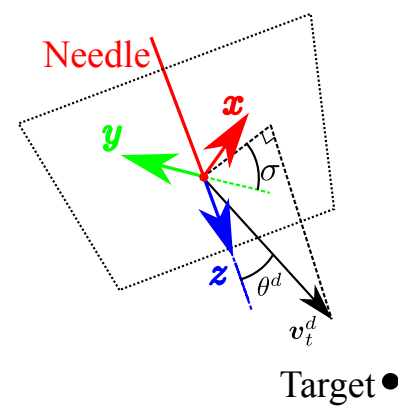

(c) Orientation angle
Fig. 1. Needle modeling

where $\Pi_{i}$ stands for the projection onto the plane $P_{i}$. The projection is used to remove the tangential part of the force and to keep only the normal part. This normal force introduces a constant shear force all along the segment, such that

$$
E I \frac{\mathrm{d}^{3} \boldsymbol{S}_{i}}{\mathrm{~d} l^{3}}(l)=-\boldsymbol{F}_{i},
$$

with $E$ the needle Young's modulus and $I$ its second moment of area. For a hollow circular needle, $I$ can be calculated from the outer and inner diameter [4], $d_{\text {out }}$ and $d_{i n}$ respectively, with the well-known formula

$$
I=\frac{\pi}{64}\left(d_{\text {out }}^{4}-d_{i n}^{4}\right) \text {. }
$$

Finally the moment due to the bevel force gives the following boundary condition:

$$
\left.E I \frac{\mathrm{d}^{2} \boldsymbol{S}_{N+1}}{\mathrm{~d} l^{2}}\right|_{l=L_{N+1}}=-M_{t i p} \boldsymbol{y}_{\text {tip }} .
$$

The above modeling provides a total of $12 \times(N+1)$ unknown variables and $12 \times(N+1)$ equations. Like in [4], it is thus possible to solve this problem as a simple linear problem.

\section{HYBRID CONTROL FOR FLEXIBLE NEEDLE}

In this section, we propose a control law that couples both base manipulation and tip based controls for the steering of the needle. At the best of our knowledge it is the first time that a method fusing these controls is proposed. The control enables the steering of the needle tip in a desired direction, while trying to reduce the tissue deformation. When the needle is aligned with the target, duty cycling allows to straighten the trajectory and reduce the needle deflection.

Let $\boldsymbol{v}_{t}^{d}$ be the desired translation velocity $\left(\operatorname{dim}\left(\boldsymbol{v}_{t}^{d}\right)=3\right)$ of the needle tip expressed in the tip frame. In practice this velocity will be given by another algorithm that generates the desired trajectory.

\section{A. Direct Base Manipulation}

Let $\boldsymbol{V}_{b}=\left[\begin{array}{ll}\boldsymbol{v}_{b} & \boldsymbol{\omega}_{b}\end{array}\right]^{T}$ be the velocity screw vector of the base, with $\boldsymbol{v}_{b}=\left[\begin{array}{lll}v_{b, x} & v_{b, y} & v_{b, z}\end{array}\right]^{T}$ being the velocity vector and $\boldsymbol{\omega}_{b}=\left[\begin{array}{lll}\omega_{b, x} & \omega_{b, y} & \omega_{b, z}\end{array}\right]^{T}$ the angular velocity vector. Similarly we define the velocity screw vector of the tip, $\boldsymbol{V}_{t}=\left[\begin{array}{ll}\boldsymbol{v}_{t} & \boldsymbol{\omega}_{t}\end{array}\right]^{T}$. Using the previously defined model, we numerically derive a manipulation Jacobian ${ }^{t} \boldsymbol{J}_{b}$. It represents a local approximation of the relation between the base and tip screw vectors given by

$$
\boldsymbol{V}_{t}={ }^{t} \boldsymbol{J}_{b} \boldsymbol{V}_{b} .
$$

The translational velocity of the tip is thus given by

$$
\boldsymbol{v}_{t}={ }^{t} \boldsymbol{J}_{b, v} \boldsymbol{V}_{b},
$$

where ${ }^{t} \boldsymbol{J}_{b, v}$ is the upper half part of ${ }^{t} \boldsymbol{J}_{b}$.

In order to reduce the tissue deformation while obtaining the desired tip trajectory, we propose to use the redundancy formalism of the task function framework [14]. This allows considering many different tasks that have to be fulfilled by the system. These tasks are hierarchically ordered and the control law is computed in such a way that low priority tasks do not induce any perturbations on higher priority tasks.

In our case, we define three tasks. A first task $e_{1}$ of dimension 3 is used to control the trajectory of the needle tip. This task has the highest priority and is defined such that $\dot{\boldsymbol{e}_{1}}=\boldsymbol{v}_{t}$. The desired behavior for this task is here $\tilde{\boldsymbol{e}}_{1}=\boldsymbol{v}_{t}^{d}$.

A second task $e_{2}$ of dimension 1 is used for the orientation of the bevel and has a medium priority. If we do not orient the bevel, additional needle bending can be necessary to compensate for the natural tip deflection. This would increase the tissue stress and is of course not desirable. To naturally reduce the tissue deformation and needle bending that is required to steer the tip in the desired direction, the bevel should face the opposite direction. In this way, the tip axis naturally orients itself toward the desired velocity vector when the needle is simply inserted. Thereby, the second task is defined as $e_{2}=\sigma$, where $\sigma$ is the angle between the current bevel orientation and the orientation given by $\boldsymbol{v}_{t}^{d}$ (see Fig. 1c), i.e.

$$
\sigma=-\frac{\pi}{2}-\operatorname{atan} 2\left(v_{t, y}^{d}, v_{t, x}^{d}\right) .
$$

We define the desired behavior of the task as $\tilde{e_{2}}=\omega_{b}$, where $\omega_{b}$ is given by a smoothed sliding mode controller [7]. This control law allows to steer $\sigma$ toward zero and is such as:

$$
\omega_{b}= \begin{cases}-\operatorname{sign}(\sigma) \omega_{z, \max } & \text { if }|\sigma| \geq \frac{\pi}{4} \\ -\frac{4}{\pi} \sigma \omega_{z, \max } & \text { if }|\sigma|<\frac{\pi}{4}\end{cases}
$$

where $\omega_{z, \max }$ is the maximum rotation speed allowed.

Finally a third task $e_{3}$ of dimension 1 is used for active reduction of the tissue deformations. We give this task the lowest priority and define it as $e_{3}=d_{m}$, where $d_{m}$ is the mean tissue displacement. This is estimated using the model proposed in section II by averaging the elongation lengths of the virtual springs

$$
d_{m}=\frac{1}{L_{\text {ins }}} \sum_{i=1}^{N} l_{i}\left\|\boldsymbol{p}_{n, i}-\boldsymbol{p}_{0, i}\right\|,
$$


where $L_{i n s}$ is the total length of the needle that is inserted in the tissue. To obtain a smooth reduction of the tissue deformation, we choose the desired behavior of the task as $\tilde{e_{3}}=-\lambda d_{m}$, where $\lambda$ is a coefficient that influences the convergence speed.

Since the optimal directions of these three tasks may be in opposition (mainly the first and third tasks), we use a singularity-robust formulation for the control law [15][16]. This way, the final control law for the needle base motion is given by

$$
\boldsymbol{V}_{b}={ }^{t} \boldsymbol{J}_{b, v}^{+} \boldsymbol{v}_{t}^{d}+\boldsymbol{P}_{1}^{t} \boldsymbol{J}_{b, \omega_{z}}^{+} \omega_{b}-\lambda \boldsymbol{P}_{12} \boldsymbol{J}_{d_{m}}^{+} d_{m},
$$

where the ${ }^{+}$symbol stands for the pseudo-inverse operator, ${ }^{t} \boldsymbol{J}_{b, \omega_{z}}$ is the sixth line of the manipulation Jacobian and $\boldsymbol{J}_{d_{m}}$ is the Jacobian of the mean tissue deformation with respect to the base screw vector. $\boldsymbol{P}_{1}$ is here the orthogonal projection matrix operator onto the null space of ${ }^{t} \boldsymbol{J}_{b, v}$ and $\boldsymbol{P}_{12}$ is the projection matrix operator onto the null space of the augmented Jacobian $\boldsymbol{J}_{12}$, namely:

$$
\begin{aligned}
\boldsymbol{J}_{12} & =\left(\begin{array}{c}
{ }^{t} \boldsymbol{J}_{b, v} \\
{ }^{t} \boldsymbol{J}_{b, \omega_{z}}
\end{array}\right), \\
\boldsymbol{P}_{1} & =\boldsymbol{I}_{6}-{ }^{t} \boldsymbol{J}_{b, v}^{+}{ }^{t} \boldsymbol{J}_{b, v}, \\
\boldsymbol{P}_{12} & =\boldsymbol{I}_{6}-\boldsymbol{J}_{12}^{+} \boldsymbol{J}_{12},
\end{aligned}
$$

where $\boldsymbol{I}_{6}$ is the $6 \times 6$ identity matrix.

\section{B. Duty Cycling Control}

The duty cycling method is used to reduce the natural curvature obtained when inserting the needle. This has been extended to 3D using different approaches in [17] and [18]. The ratio between the natural curvature $K_{n a t}$ of the needle and the effective curvature $K_{\text {eff }}$ has been shown in [9] to be linearly dependent on the duty cycle $D C$, i.e. the ratio between the two phases durations:

$$
K_{\text {eff }}=K_{\text {nat }}(1-D C)
$$

On a kinematics point of view, $D C$ actually corresponds to the ratio between the insertion length of the phases. In our case, we define a fixed insertion length $L_{D C}$ for the cycles and allow the use of different insertion speeds for the two phases. This way we can avoid the infinite cycle duration obtained when $D C$ goes to zero as stated in [18].

We use duty cycle control when the desired tip velocity $\boldsymbol{v}_{t}^{d}$ is almost aligned with the needle tip axis. This is detected by comparing the angle $\theta^{d}$ between $\boldsymbol{v}_{t}^{d}$ and the tip axis (Fig. 1c) with the angle $\theta^{D C}$ that would be obtained during a cycle with duty cycle equal to zero, i.e. with an insertion phase only. $\theta^{d}$ and $\theta^{D C}$ are expressed as

$$
\begin{gathered}
\theta^{d}=\operatorname{atan} 2\left(\sqrt{{v_{t, x}^{d}}^{2}+v_{t, y}^{d}{ }^{2}}, v_{t, z}^{d}\right), \\
\theta^{D C}=K_{n a t} L_{D C} .
\end{gathered}
$$

If $\theta^{d}<\theta^{D C}$, it means that the needle would overshoot the current desired direction in less than a cycle length. In that case it is better to reduce the effective curvature of the needle such that it aligns with the desired direction, i.e with

$$
K_{e f f}=\frac{\theta^{d}}{L_{D C}} .
$$

Using the same approach as in [18], we also define an accumulation angle for the rotation around the tip axis equal to $\sigma$ (see eq. (14)), such that the bevel is in the adequate direction before starting the translation phase.

Finally, since the needle can be bent, the optimal insertion direction of the base $\boldsymbol{V}_{b, \text { ins }}$ may be different from the needle base axis. Here we choose to compute it as the base motion that maximizes the tip insertion in the axial direction, $\boldsymbol{V}_{b, i n s}={ }^{t} \boldsymbol{J}_{b, v_{z}}^{+}$, where ${ }^{t} \boldsymbol{J}_{b, v_{z}}$ is the third line of the manipulation Jacobian.

\section{EXPERIMENTAL RESULTS}

In this section we present the experimental setup used to validate the control method and the results.

\section{A. Experimental setup}

A needle is fixed to the end effector of a 6 DOFs Adept Viper S650 robot. We use a stainless steel biopsy needle with internal and external diameters of $0.48 \mathrm{~mm}$ and $0.7 \mathrm{~mm}$ respectively. The length of the needle that can bend, i.e. the part that is outside the needle holder, is $12.6 \mathrm{~cm}$ long. The needle Young's modulus is assumed to be that of steel, i.e. $200 \mathrm{GPa}$. The insertion is done in a homemade gelatin phantom embedded in a transparent plastic container. For a validation purpose, the visual feedback is here obtained using two optical cameras. The needle is detected and tracked in real time by an image processing algorithm that we briefly introduce in the following. A white screen monitor is used to provide some light in the background and enables the observation of the needle through the gelatin. Figure $2 \mathrm{e}$ shows a picture of this experimental setup. The intrinsic parameters of both cameras were calibrated as well as the extrinsic parameters between the cameras. In this way, stereo-vision is used to retrieve the $3 \mathrm{D}$ position of the needle observed in both camera images. The accuracy of this tracking system, corresponding to the size of the pixels, is around $0.25 \mathrm{~mm}$. Before the experiments, the gelatin container was removed and the shape of the needle was detected. As the position of the needle is known with respect to the robot, the transformation between the robot frame and the stereo-vision system was estimated. Note that we plan in the future to replace this visual system by a 3D ultrasound probe, thus providing directly a 3D position without the need for stereo-vision calibration.

\section{B. Model update from visual feedback}

The pose of the needle base in the model is updated using the odometry of the robot and the fact that the orientation of the bevel with respect to the robot is known at the beginning of the procedure. The needle is tracked in each image and the 3D shape of the needle that is visible in both images is computed. As the needle is clearly visible in the images, 


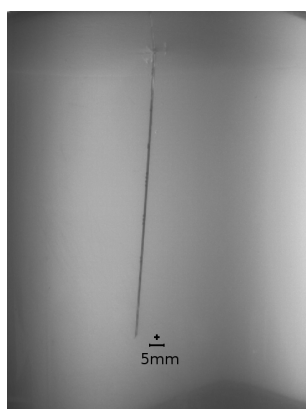

(a) No control, target is aligned

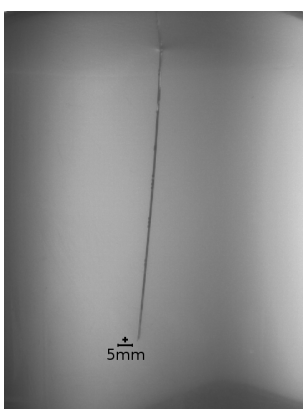

(b) No control, target is shifted

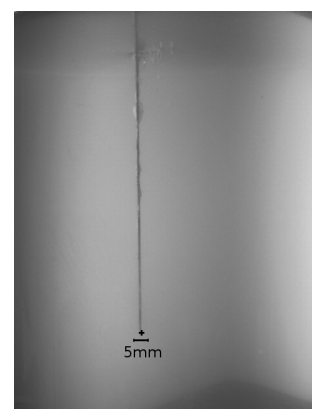

(c) Control is active, target is aligned

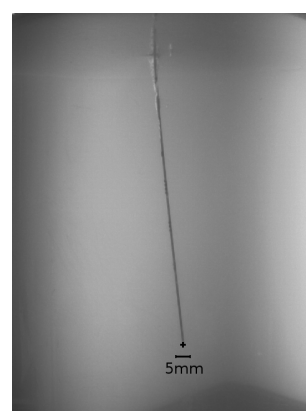

(d) Control is active, target is shifted

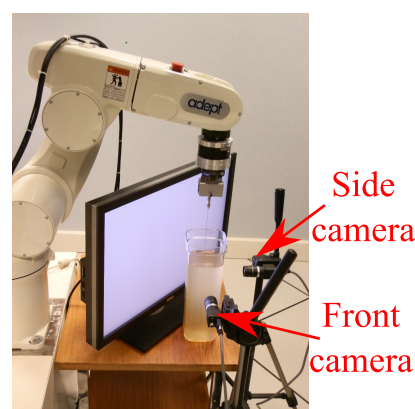

(e)

Fig. 2. Final view of the front camera at the end of the experiments. The crosses represent the target. Refer to the accompanying video for real time view of the insertions. (e) Experimental setup

we use here a simple tracking algorithm that consists in estimating the needle shape with a third order polynomial defined by four control points. The position of the control points is updated and filtered with a Kalman filter at each image acquisition such that they follow the needle shape and its tip. We don't describe the algorithm further here because it is not the subject of this paper and it should be adapted to a more relevant imaging modality in the future.

To have a good modeling of the tissue, virtual springs must be regularly added all along the modeled needle shaft. We choose here to add a new spring when the length of the last needle segment reaches a fixed threshold of $2.5 \mathrm{~mm}$. When adding a spring at the needle tip, we define the rest position of the spring at the location of the tip. This way the rest position represents the initial location of the tissue when it is just being cut by the bevel. To fit the model to the reality, this position is obtained from the real needle tip using the visual feedback.

The model also requires some parameters as inputs. The parameters related to the needle, such as its geometry and Young's modulus, are already known and should not vary significantly during the insertion procedure. The tissue stiffness per unit length $K_{T}$, however, must be estimated. We propose here to do an online estimation of this parameter. The best value is computed at each image acquisition using a least square approach such that the shape of the model needle fits the measured needle shape.

\section{Experiments}

Four experiments were performed to validate our method. The stiffness per unit length of the model was initialized in each case with $K_{T}=10000 \mathrm{~N} / \mathrm{m}^{2}$. At the beginning of each experiment, the needle is placed such that it is normal to the surface of the gelatin and its tip slightly touches it. The insertion point is shifted between the experiments in a way that the needle can not cross a previous insertion path. The needle is first inserted along a distance of few millimeters in the gelatin to allow the manual initialization of the tracking algorithm in the images. Then the insertion procedure is started and is stopped when the target is not in front of the needle tip anymore.
In a first experiment, a virtual target is defined before the beginning of the insertion such that it is aligned with the needle and placed at a distance of $8 \mathrm{~cm}$ from the tip. An insertion is then performed without activating the controller at an insertion speed of $1 \mathrm{~mm} / \mathrm{s}$, i.e. the needle is simply inserted along its shaft direction. The insertion is stopped when the needle tip passes the target location. Fig. 2a shows the view of the front camera at the end of the experiment. We can see that the target is missed due to the natural deflection of the needle. Fig. 3a shows the 3D lateral distance between the needle tip axis and the target. It can be seen that the needle passes almost $8 \mathrm{~mm}$ away from the target at the end of the insertion.

In a second experiment, the designed controller is activated. The input velocity vector $\boldsymbol{v}_{t}^{d}$ for the controller is chosen such that it is always pointing toward the target and has a norm of $1 \mathrm{~mm} / \mathrm{s}$ :

$$
\boldsymbol{v}_{t}^{d}=0.001 \frac{\boldsymbol{p}_{\text {tar }}}{\left\|\boldsymbol{p}_{\text {tar }}\right\|},
$$

with $\boldsymbol{p}_{\text {tar }}$ the position of the target in the tip frame, deduced from the visual feedback. As can be seen on Fig. 3c the controller allows to reach the target with a sub-millimeter accuracy, thus validating the framework.

Two other experiments were performed similarly to the previous ones, except that the target is shifted $1 \mathrm{~cm}$ away from the initial tip axis. For the insertion without the controller (third experiment), the target is placed in the bevel direction (opposite of the $y$ axis on Fig.1b) such that the needle naturally bends toward the target when inserted. For the last experiment, the controller is activated and the target is placed to forms an angle of 135 degrees with the bevel direction to be sure that the controller has to rotate the needle to align the bevel with the target.

We can see on figure Fig. $2 b$ that the target can not be reached when the controller is not active. Fig. $3 b$ shows that the $3 \mathrm{D}$ lateral distance between the target and the tip axis tends to decrease but the target is finally missed by $5 \mathrm{~mm}$. A pure insertion actually corresponds to a saturated duty cycling control with duty cycle $D C$ equal to 0 . Using only the natural tip deflection to control the needle is thus not enough for this targeting task because of the low curvature of 
TABLE I

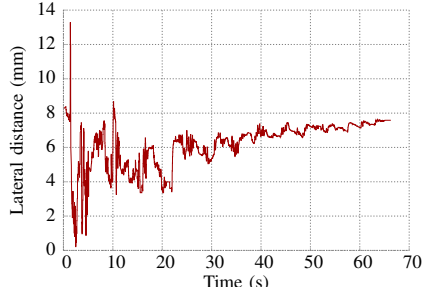

(a) No control and aligned target

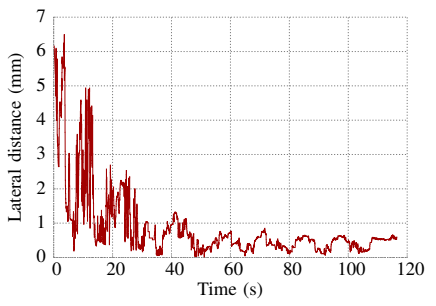

(c) Active control and aligned target

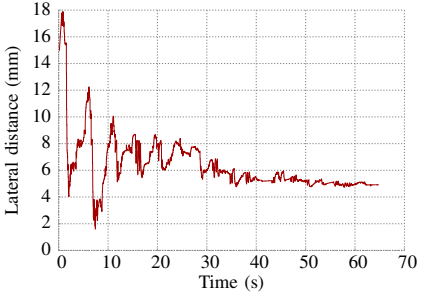

(b) No control and shifted target

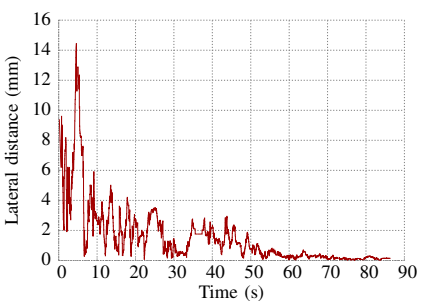

(d) Active control and shifted target
Fig. 3. Measure of the 3D lateral distance between the needle tip axis and the target. Note that the measure is noisier at the beginning, since errors on the measure of the tip axis orientation are amplified when the target is far from the tip.

the needle. Since our controller uses additional base motions, it allows to reach the target as can be seen on Fig. 2c and 2 d. Fig. $3 d$ shows that the target could also be reached with sub-millimeter accuracy. This way the designed controller allows to increase the reachable space compared to the sole use of tip-based control methods. Table I shows a summary of the final lateral targeting error between the tip and the target. Note that the axial error (in the needle direction) was under $0.2 \mathrm{~mm}$ for each experiment, which corresponds to the accuracy of the vision system.

\section{CONCLUSIONS AND FUTURE WORK}

In this paper we proposed a new control method for flexible needle steering that combines direct base manipulation and tip based control methods. The direct base manipulation control is generated thanks to the use of a 3D model of a beveled tip needle that gives the adequate motion of the needle base to obtain a given motion of the tip. An update of the model is performed online using visual feedback. The duty cycling method is jointly used to reduce the needle natural curvature and to obtain relatively straight trajectories when needed. We observed that our controller allows to obtain a good targeting accuracy and is able to reach targets that are not reachable using only tip-based control.

As a next step, the cameras will be replaced by 3D ultrasound probe to fit with the clinical context. The tracking algorithm should be adapted for ultrasound images, as for example by using the one presented in [19]. One drawback with the current formulation of the control law is that little deformation reduction is achieved when the task comes in opposition with the targeting task. Least squares optimization could be used to find a better control, but at the expense of greater computation load. We also plan to extend the update of the model to take into account the displacements of the tissues that are due to the physiological motion of the patient.
FINAL LATERAL ERROR in MM

\begin{tabular}{|c|c|c|}
\cline { 2 - 3 } \multicolumn{1}{c|}{} & No control & Active control \\
\hline Aligned target & 7.6 & 0.6 \\
\hline Shifted target & 4.9 & 0.1 \\
\hline
\end{tabular}

\section{REFERENCES}

[1] K. Reed, A. Majewicz, V. Kallem, R. Alterovitz, K. Goldberg, N. Cowan, and A. Okamura, "Robot-assisted needle steering," Robotics Automation Magazine, IEEE, vol. 18, pp. 35-46, Dec 2011.

[2] R. Webster III, J. Kim, N. Cowan, G. Chirikjian, and A. Okamura, "Nonholonomic modeling of needle steering," The International Journal of Robotics Research, vol. 25, no. 5-6, pp. 509-525, 2006.

[3] S. DiMaio and S. Salcudean, "Needle steering and motion planning in soft tissues," Biomedical Engineering, IEEE Transactions on, vol. 52, pp. 965-974, June 2005.

[4] D. Glozman and M. Shoham, "Image-guided robotic flexible needle steering," Robotics, IEEE Transactions on, vol. 23, pp. 459-467, June 2007.

[5] Z. Neubach and M. Shoham, "Ultrasound-guided robot for flexible needle steering," Biomedical Engineering, IEEE Transactions on, vol. 57, pp. 799-805, April 2010.

[6] A. Okamura, C. Simone, and M. O'Leary, "Force modeling for needle insertion into soft tissue," Biomedical Engineering, IEEE Transactions on, vol. 51, pp. 1707-1716, Oct 2004.

[7] D. Rucker, J. Das, H. Gilbert, P. Swaney, M. Miga, N. Sarkar, and R. Webster, "Sliding mode control of steerable needles," Robotics, IEEE Transactions on, vol. 29, pp. 1289-1299, Oct 2013.

[8] K. Hauser, R. Alterovitz, N. Chentanez, A. Okamura, and K. Goldberg, "Feedback control for steering needles through $3 \mathrm{~d}$ deformable tissue using helical paths," Robotics science and systems: online proceedings, p. 37,2009

[9] D. Minhas, J. Engh, M. Fenske, and C. Riviere, "Modeling of needle steering via duty-cycled spinning," in Engineering in Medicine and Biology Society, 2007. EMBS 2007. 29th Annual International Conference of the IEEE, pp. 2756-2759, Aug 2007.

[10] S. Patil, J. Burgner, R. Webster, and R. Alterovitz, "Needle steering in 3-d via rapid replanning," Robotics, IEEE Transactions on, vol. 30, pp. 853-864, Aug 2014.

[11] P. Moreira, S. Patil, R. Alterovitz, and S. Misra, "Needle steering in biological tissue using ultrasound-based online curvature estimation," in Robotics and Automation (ICRA), 2014 IEEE International Conference on, pp. 4368-4373, May 2014.

[12] S. Misra, K. Reed, B. Schafer, K. Ramesh, and A. Okamura, "Mechanics of flexible needles robotically steered through soft tissue," The International Journal of Robotics Research, 2010.

[13] M. Abayazid, R. Roesthuis, R. Reilink, and S. Misra, "Integrating deflection models and image feedback for real-time flexible needle steering," Robotics, IEEE Transactions on, vol. 29, pp. 542-553, April 2013.

[14] B. Siciliano and J.-J. Slotine, "A general framework for managing multiple tasks in highly redundant robotic systems," in Advanced Robotics, 1991. 'Robots in Unstructured Environments', 91 ICAR., Fifth International Conference on, pp. 1211-1216 vol.2, June 1991.

[15] S. Chiaverini, "Singularity-robust task-priority redundancy resolution for real-time kinematic control of robot manipulators," Robotics and Automation, IEEE Transactions on, vol. 13, pp. 398-410, Jun 1997.

[16] N. Mansard and F. Chaumette, "Task sequencing for high-level sensorbased control," Robotics, IEEE Transactions on, vol. 23, pp. 60-72, Feb 2007.

[17] N. Wood, C. Lehocky, and C. Riviere, "Algorithm for threedimensional control of needle steering via duty-cycled rotation," in Mechatronics (ICM), 2013 IEEE International Conference on, pp. 237-241, Feb 2013.

[18] A. Krupa, "A new duty-cycling approach for 3d needle steering allowing the use of the classical visual servoing framework for targeting tasks," in 5th IEEE RAS/EMBS International Conference on Biomedical Robotics and Biomechatronics, BioRob, (Sao Paulo, Brazil), pp. 301-307, August 2014.

[19] P. Chatelain, A. Krupa, and N. Navab, "3d ultrasound-guided robotic steering of a flexible needle via visual servoing," in Robotics and Automation (ICRA), 2015 IEEE International Conference on, (Seattle, WA), pp. 2250-2255, May 2015. 